\title{
HIGH RESOLUTION INTERFEROMETRY OF COOL STARS
}

\author{
D. L. LA MBERT \\ McDonald Observatory \\ and \\ Dept. of Astronomy, The University of Texas at Austin, Austin, Tex., U.S.A.
}

\begin{abstract}
This paper reviews results obtained in a program of high resolution infrared spectroscopy of $\mathrm{K}$ and $\mathrm{M}$ giants. The reported results include (i) a preliminary analysis of the $\mathrm{C}, \mathrm{N}$ and $\mathrm{O}$ abundances for $\alpha$ Ori, (ii) determinations of the ${ }^{12} \mathrm{C} /{ }^{13} \mathrm{C}$ and ${ }^{16} \mathrm{O} /{ }^{18} \mathrm{O}$ ratios for $\alpha$ Ori ${ }^{12} \mathrm{C} /{ }^{13} \mathrm{C} \sim 6$ and ${ }^{16} \mathrm{O} /{ }^{18} \mathrm{O} \sim 500$ ), (iii) the derivation of a lower limit, ${ }^{16} \mathrm{O} /{ }^{18} \mathrm{O} \approx 300$, for the ${ }^{16} \mathrm{O} /{ }^{18} \mathrm{O}$ ratio in $\alpha$ Boo, (iv) a discussion of observations of the $\mathrm{SiO}$ first-overtone vibration-rotation bands in $\mathrm{M}$ stars, and (v) a search for the fundamental quadrupole vibration-rotation lines of $\mathbf{H}_{2}$.
\end{abstract}

\section{Introduction}

This paper describes a selection of the results obtained in a program of infrared high resolution spectroscopy of cool stars. This program is a collaborative venture between University of Texas astronomers and Dr Reinhard Beer of the Jet Propulsion Laboratory in Pasadena, California.

The high resolution spectra are obtained with a Connes-type Fourier transform spectrometer located at the coudé focus of the McDonald Observatory's 107-in. reflector. This instrument was constructed at the Jet Propulsion Laboratory and a full description is available (Beer et al., 1971). The highest resolution attainable is 0.03 $\mathrm{cm}^{-1}$. Presently available stellar spectra correspond to a resolution of $0.1 \mathrm{~cm}^{-1}$ for the brightest objects. The useful spectral range is either 1.4 to $2.3 \mu$ or 3.0 to $5.0 \mu$.

The goal of the observing program is to obtain spectra at a resolution of about $0.1 \mathrm{~cm}^{-1}$ for bright infrared stars; the working list includes giants and supergiants of spectral types $\mathrm{K}$ and $\mathrm{M}$, long period variables and carbon stars. Since the observing time required to achieve a certain signal-to-noise ratio is inversely proportional to the square of the resolution, a compromise must be reached between resolution and signal-to-noise ratio. The desirable resolution depends on the star and the problems under investigation. The following example illustrates this problem. The absorption lines of M-type supergiants (e.g. $\alpha$ Ori, $\alpha$ Sco) are very broad. A direct interpretation is that the atmospheres are very turbulent with velocities of the order of $10 \mathrm{~km} \mathrm{~s}^{-1}$. If the velocity distribution is Gaussian with a full halfwidth at half maximum (FWHM) of $10 \mathrm{~km} \mathrm{~s}^{-1}$, a weak absorption line has a profile with a FWHM of $0.2 \mathrm{~cm}^{-1}$ at $6000 \mathrm{~cm}^{-1}(1.7 \mu)$ or $0.1 \mathrm{~cm}^{-1}$ at $3000 \mathrm{~cm}^{-1}(3.3 \mu)$. In this example, a resolution of $0.1 \mathrm{~cm}^{-1}$ in the 1.4 to $2.3 \mu$ atmospheric windows will yield a spectrum which is not seriously degraded by the instrumental profile. Furthermore, the instrumental profile is well defined so that a correction for instrumental smearing may be applied. Line widths and turbulent motions are substantially smaller for the 
typical $\mathrm{K}$ giant star and a resolution of better than $0.1 \mathrm{~cm}^{-1}$ appears necessary in order to fully resolve the line spectrum.

\section{The Nature of Infrared Stellar Spectra}

Infrared stellar spectra contain classes of transitions which are sparsely represented in the previously analyzed visible spectra. An outline of the general nature of the infrared spectra is sketched here.

Low resolution infrared spectra of cool stars are dominated by the $\mathrm{R}$ branch heads of the $\mathrm{CO}$ vibration-rotation transitions. Although other molecules may not provide so spectacular a contribution, vibration-rotation transitions of molecules composed of the astrophysically abundant elements provide a rich and vital contribution to the spectra. The fundamental series of bands for the lighter molecules are located in or near the 3 to $5 \mu$ atmospheric windows. Overtones for the abundant molecules can be detected at shorter wavelengths; for example, the 1.4 to $2.3 \mu$ interval includes the first and second overtone bands of $\mathrm{CO}$ and the first-overtone bands of the $\mathrm{OH}$ radical. Heavier molecules have their fundamental transitions at longer wavelengths; SiO has first overtone bands near $4 \mu$. Vibration-rotation bands have not been detected in the visible region of stellar spectra; high order overtones would be necessary but these are intrinsically very weak. All of the presently observed transitions involve the ground electronic state of a molecule.

In the visible region, molecules contribute to the line spectrum through electronic transitions. In general, the separations of the lowest electronic states in molecules (also, in atoms) are such that transitions between them are located in the visible or ultraviolet. However, a few electronic transitions do contribute to the infrared stellar spectra. Examples are the $\mathrm{CN}$ red system and the Phillips and Ballik-Ramsay systems of $\mathrm{C}_{2}$.

The contribution from atoms to the stellar infrared spectra is substantial. As the wavelength of observation increases, the average excitation potential of the contributing lines must increase. This result is a reflection of the fact that infrared transitions requiring energy level spacings of a few thousand wavenumbers must occur relatively near the ionization potential of the atom or ion. A typical infrared line of Si I has a lower state excitation potential of about $6 \mathrm{eV}$. This rather high value might prompt an expectation that the lines should be weaker in cool stars than in the Sun. In general, this expectation is not fulfilled because the increased scale heights for the luminous cool stars can compensate for the reduced excitation at the lower temperatures: weak lines in the solar spectrum appear as strong lines in the spectrum of an $M$ type supergiant. The complex rare-earths can provide infrared transitions of low excitation potential.

A comment on the continuum radiation is not out of place among these general remarks on the spectrum. A typical cool star is now recognized to have a circumstellar shell which is detectable through the infrared excess across the 8 to $13 \mu$ atmospheric window. In extreme cases, this shell may contribute to the spectrum at shorter wavelengths and produce a dilution of the photospheric lines. 


\section{Astrophysics and Stellar Infrared Spectroscopy}

Detailed analyses of infrared spectra of cool stars will provide answers to many astrophysical problems. Two general problem areas are

- the physics of the stellar atmospheres

- cool stars and problems of stellar evolution.

The atmospheres of evolved cool stars represent a fierce challenge to the astrophysicist. The following selection should suffice to demonstrate the wealth of problems.

- The low gas density in giant and supergiant atmospheres provides near optimum conditions for the appearance of departures from local thermodynamic equilibrium. These departures must be considered in abundance analyses and in the treatment of atomic and molecular line blanketing in model atmosphere construction.

- The atmospheres appear to be very turbulent. Supergiants like $\alpha$ Ori and $\alpha$ Sco have very broad absorption lines which may correspond to supersonic turbulent velocities. The velocity fields have to be defined and their influence on the atmosphere evaluated; the turbulence will provide a pressure term in the photosphere and, also, provide a source of mechanical energy for chromospheric heating.

- A substantial chromosphere has been inferred from observations of strong Ca II $\mathrm{H}$ and $\mathrm{K}$ emission. The chromospheric structure is unknown. The possibility of a corona has not been definitively excluded. These particular questions may be answered from searches for the ultraviolet emission lines from the chromospheric-coronal gas. The infrared studies should provide the photospheric boundary conditions including estimates for the mechanical energy reservoir.

- Circumstellar shells are detected by the infrared excess and the narrow violetdisplaced absorption components which accompany resonance lines of atoms and ions. Mass-loss rates have been estimated from analyses of the expansion rates for the shell. The physics of the shell is incomplete; for example, the origin of the expansion is presently uncertain.

- The outer atmosphere may be a site for dust grain formation. The influence of grains on the atmosphere has to be explored fully.

The stellar evolutionary steps which transfer a main sequence star to the red giant region are moderately well understood. A detailed mapping of evolution during the red giant stage is beginning and feedback from observational programs is likely to prove very productive.

In many applications of spectroscopic abundance analyses, the assumption often implicit - is made that the surface composition is representative of the initial composition of the star. This is an invalid assumption for the cool evolved stars. Their initial surface layers may be mixed with processed material from the interior. Convective mixing can occur as the star evolves up the red giant branch and a more violent mixing is predicted to occur at the helium flash stage. No doubt, other possibilities may become apparent as theoretical efforts are expanded in this area. Direct observational checks are needed. The key elements and their isotopes are $\mathrm{H}, \mathrm{He}, \mathrm{Li}, \mathrm{C}, \mathrm{N}$ and $\mathrm{O}$. The abundant elements, $\mathrm{C}, \mathrm{N}, \mathrm{O}$ and $\mathrm{H}$ provide molecules with vibration-rotation transi- 
tions in the infrared. The frequency of a vibration-rotation transition is dependent on the masses of the vibrating -rotating nuclei and an isotopic substitution can give a readily detectable frequency shift. The important ${ }^{12} \mathrm{C} /{ }^{13} \mathrm{C}$ ratio is an example of a quantity which can be extracted from the infrared spectra and which is a measure of the mixing experienced by the star.

\section{An Abundance Analysis for $\propto$ Ori (Betelgeuse)}

Calculations based upon single slab models (Spinrad et al., 1970; Beer et al., 1972) had suggested that the present composition represented strong evidence for abundance changes wrought by mixing: i.e. $\mathrm{C}$ was underabundant by a large factor and $\mathrm{N}$ was overabundant. These results have been reexamined using a model atmosphere. The full analysis will be published elsewhere.

Model atmospheres for $\alpha$ Ori were described by Faÿ and Johnson (1973). The abundance calculations are based upon a model kindly supplied by Professor Hollis Johnson. Calculations of the absorption line profiles are based on the customary set of assumptions: local thermodynamic equilibrium, plane-parallel homogeneous layers and hydrostatic equilibrium. All of these assumptions deserve a critical examination when applied to an $M$ supergiant atmosphere.

The initial goal was to derive abundances for the light elements $\mathrm{C}, \mathrm{N}$ and $\mathrm{O}$. The spectral features of relevance are

- $\mathrm{CO}$ : the fundamental, first and second overtone vibration-rotation bands

- OH: The fundamental and first-overtone vibration-rotation bands

- CN: the red $\left(A^{2} I I-X^{2} \Sigma\right)$ system

- SiO: the first-overtone $(4 \mu)$ vibration-rotation bands.

These transitions have been identified on the infrared spectra. In addition, the 2-0 band of the $\mathrm{CN}$ red system near $8000 \AA$ has been observed with a photoelectric coudé scanner. This scanner is also being used to observe permitted and forbidden lines of $C$ and $O$ in the visible and near infrared.

The analysis procedure is approximately as follows:

- the $\mathrm{CO}$ line intensities provide an estimate for the $\mathrm{C}$ abundance $(\mathrm{C}$ is associated into $\mathrm{CO}$ throughout the greater part of the atmosphere).

- the $\mathrm{OH}$ provides the $\mathrm{O}$ (in effect, $\mathrm{O}-\mathrm{C}$ ) abundance.

- the $\mathrm{CN}$ gives the $\mathrm{N}$ abundance when the $\mathrm{C}$ is known.

The available infrared spectra correspond to a resolution of $0.1 \mathrm{~cm}^{-1}$. The interval $5500-6600 \mathrm{~cm}^{-1}$ is available at a signal-to-noise ratio of about 60 to 1 . The 3 to $5 \mu$ region spectrum has a rather poor signal-to-noise ratio of 15 to 1 . A spectrum of the 3 to $4 \mu$ region (Beer et al., 1972) is available at a resolution of $0.29 \mathrm{~cm}^{-1}$ and a signalto-noise ratio of 150 to 1 .

The $\mathrm{CO}$ fundamental and first-overtone lines are predominantly highly saturated. The abundance analysis is based on the relatively unsaturated second-overtone lines near $1.6 \mu$. The oscillator strengths were computed from matrix elements provided by Young and Eachus (1966) which are based on room temperature measurements of 

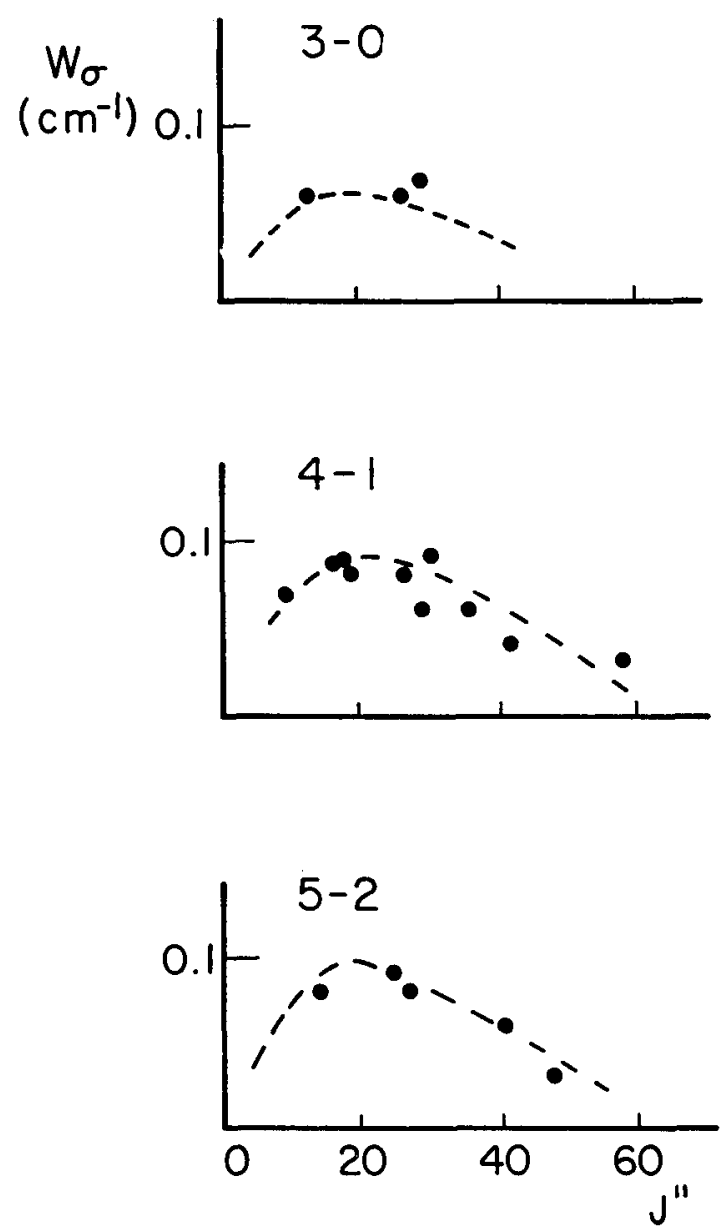

Fig. 1. Equivalent widths for $P$ branch members of the ${ }^{12} \mathrm{C}^{16} \mathrm{O}$ second-overtone bands $3-0,4-1$ and 5-2 in the spectrum of $\alpha$ Ori. The predictions (dashed lines), which are fitted to the observations (filled circles), are described in the text.

the absorption coefficients. The equivalent width for single unblended lines was measured and model atmosphere predictions were fitted to these observations (Figure 1). The $R$ branch band heads were synthesized. Observed and predicted band heads for four bands are illustrated in Figures 2 to 5 . The predicted profiles correspond to a reduction in the carbon abundance by a factor of two relative to the solar value. Oxygen was assumed to have a solar abundance.

The $\mathrm{OH}$ fundamental vibration-rotation bands in $\alpha$ Ori were discovered by Beer $e t$ al. (1972). First-overtone lines near $1.6 \mu$ are prominent. The four $P(11) 2-0$ lines are shown in Figure 6. The fundamental lines and the strongest first-overtone lines are subject to saturation effects. The absolute oscillator strengths for the $1-0$ and 2-1 bands were measured by d'Incan et al. (1971). Since relative transition probabilities 


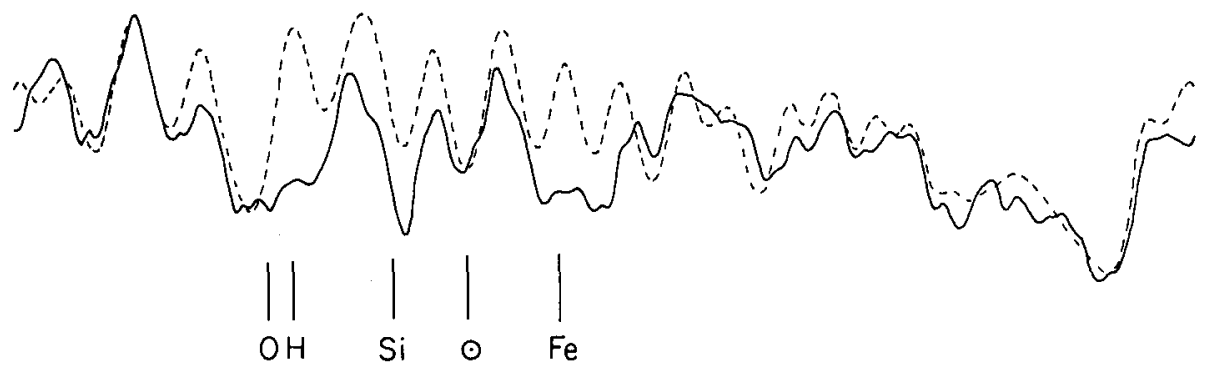

6410.0

6415.0

\section{WAVENUMBER}

Fig. 2. The $R$ branch head of the $3-0{ }^{12} \mathrm{C}^{16} \mathrm{O}$ vibration-rotation band in the spectrum of $\alpha$ Ori. The synthesized spectrum (dashed line) is fitted to the observed spectrum (solid line). The former includes the $\mathrm{CO}$ and $\mathrm{CN}$ lines but not the $\mathrm{OH}$ and atomic lines identified below the spectrum.

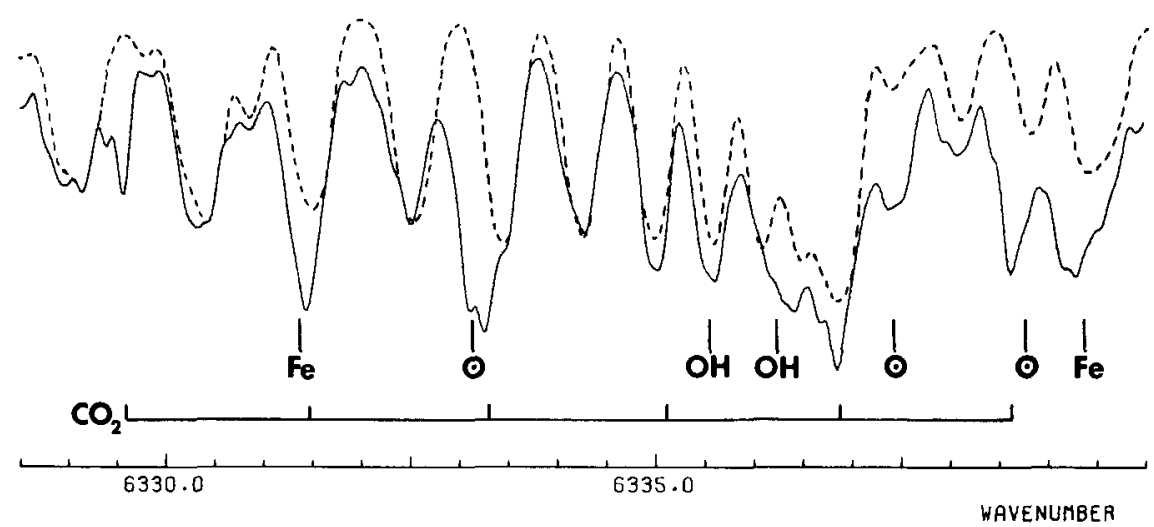

Fig. 3. The $R$ branch head of the 4-1 ${ }^{12} \mathrm{C}^{16} \mathrm{O}$ vibration-rotation band in the spectrum of $\alpha$ Ori (see caption to Figure 2). Telluric $\mathrm{CO}_{2}$ lines are identified: their narrow profiles are to be contrasted with the broad stellar line profiles.

for fundamental and first-overtone bands were measured by Murphy (1971), absolute oscillator strengths for the first-overtone bands may be obtained. The model atmosphere predictions suggest that a factor of two increase in the oxygen abundance is required to explain the observed line intensities. A careful error analysis will have to be made before the oxygen overabundance is clearly established. In particular, the $\mathrm{OH}$ lines are gravity and temperature sensitive; modest decreases in surface gravity or effective temperature would remove the required overabundance.

The $\mathrm{CN}$ analysis is presently based on the observations of the 2-0 band near $8000 \AA$. The photoelectric scans were obtained with the coudé scanner at the 107 -in. reflector (Tull, 1972). The resolution is about $0.1 \AA$ and a single scan covers approxi- 


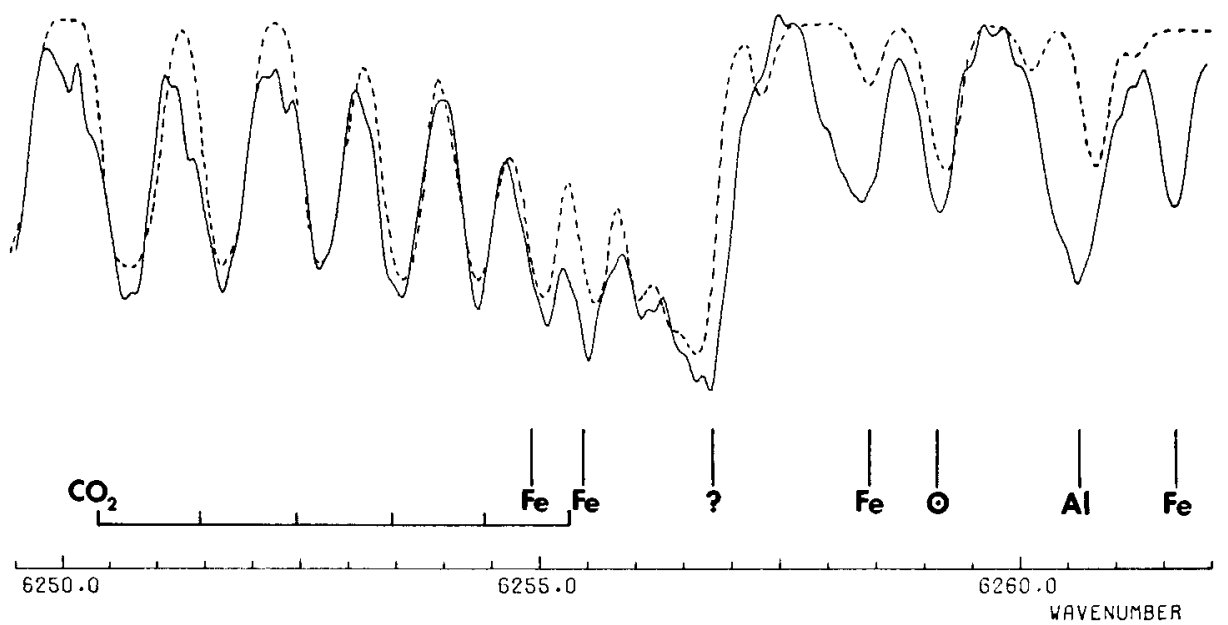

Fig. 4. The $R$ branch head of the ${ }^{5-2}{ }^{12} \mathrm{C}^{16} \mathrm{O}$ vibration-rotation band in the spectrum of $\alpha \mathrm{Or}^{\mathrm{i}}$ (see caption to Figure 2).

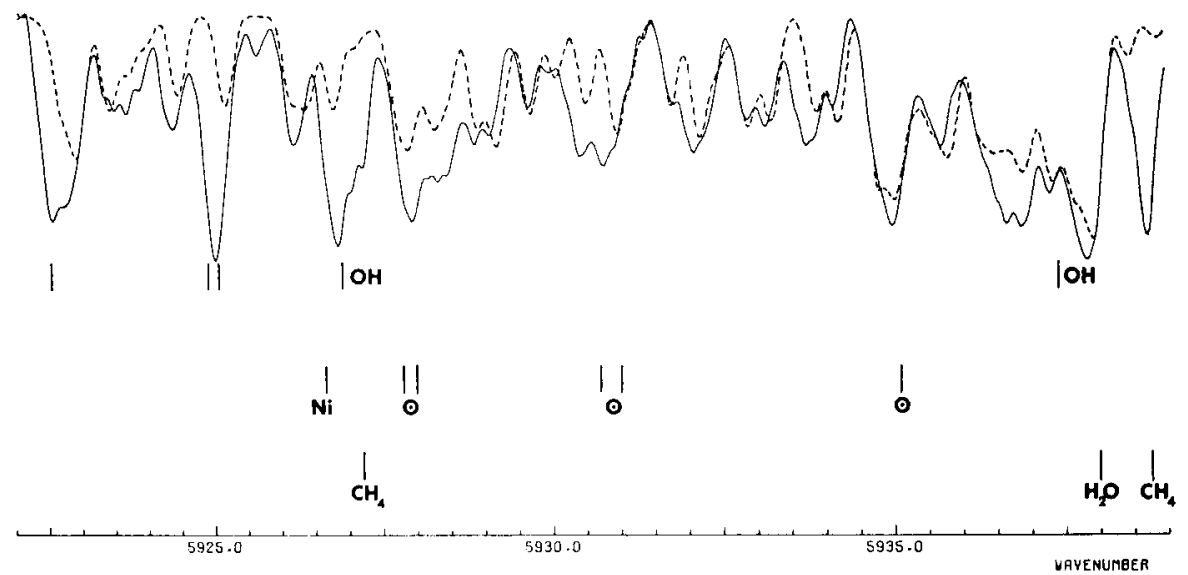

Fig. 5. The $R$ branch head of the $9-6{ }^{12} \mathrm{C}^{16} \mathrm{O}$ vibration-rotation band in the spectrum of $\alpha$ Ori (see caption to Figure 2). Telluric $\mathrm{H}_{2} \mathrm{O}$ and $\mathrm{CH}_{4}$ lines are identified.

mately $30 \AA$. A difficulty arises in the choice of dissociation energy and oscillator strength. Several recent independent experiments (see a summary by Arnold and Nicholls, 1973) have led to a preferred dissociation energy $D_{\mathrm{o}}=7.85 \mathrm{eV}$. However, this value with the measured oscillator strengths leads to predicted solar $\mathrm{CN}$ line intensities which are inconsistent with the observed intensities for both the violet $\left(B^{2} \Sigma-X^{2} \Sigma\right)$ and the red $\left(A^{2} \Pi-X^{2} \Sigma\right)$ systems (Lambert and Mallia, 1974). This discrepancy is approximately a factor of two. The origin of this discrepancy is unclear. This preliminary analysis for $\alpha$ Ori is based on the dissociation energy $D_{0}=7.85 \mathrm{eV}$ and the oscillator strength for the $0-0$ band which is required to fit the observed solar equivalent widths $\left(f_{00}=1.0 \times 10^{-3}\right)$. Relative band oscillator strengths $\left(f_{V^{\prime} V^{\prime \prime}} / f_{00}\right)$ 


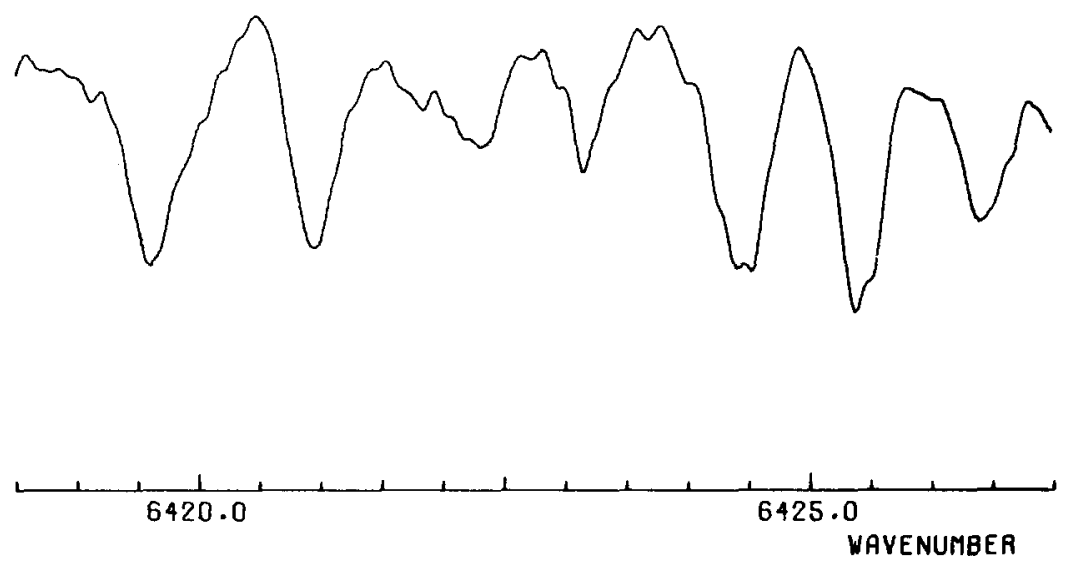

Fig. 6. First-overtone vibration-rotation lines of the hydroxyl $(\mathrm{OH})$ radical in the spectrum of the $\alpha$ Ori: the $P(11) 20$ lines are at $6419.6,6420.9,6424.4$ and $6425.4 \mathrm{~cm}^{-1}$ in this spectrum.

were taken from Arnold and Nicholls (1972). An alternative procedure would be to adopt $D_{0}=7.5 \mathrm{eV}$ and to use the measured oscillator strength $\left(f_{00}=3.4 \times 10^{-3}\right)$. This would lead to a slightly higher nitrogen abundance. With the higher dissociation energy, the model atmosphere calculations can be fitted to the observations with an approximately solar nitrogen abundance.

The results of the preliminary abundance analysis show that the $\mathrm{C}, \mathrm{N}$ and $\mathrm{O}$ abundances have their solar values to within a factor of two. This contradicts results of earlier analyses which showed considerable departures from solar values. These earlier analyses were based on a simple slab model for the atmosphere. The CO firstovertone and the $\mathrm{OH}$ fundamental lines were analyzed and both are severely saturated.

The preliminary analysis described here will be extended to include permitted and forbidden lines of $\mathrm{O}_{\mathrm{I}}$ and $\mathrm{C}$ I. The effects of changes in the model atmosphere parameters will also be studied.

\section{The ${ }^{12} \mathrm{C} /{ }^{13} \mathrm{C}$ Abundance Ratio}

As material processed in the interior is brought to the surface, the ${ }^{12} \mathrm{C} /{ }^{13} \mathrm{C}$ ratio will change. An increase in ${ }^{13} \mathrm{C}$ is anticipated. The terrestrial ratio is ${ }^{12} \mathrm{C} /{ }^{13} \mathrm{C}=89$. If the ratio for a stellar atmosphere can be shown to be significantly different, the mixing hypothesis would be confirmed and quantitative tests can be attempted. If certain conditions are satisfied, an isotopic abundance ratio can be measured with substantially greater accuracy than an element abundance. The ideal situation involves the comparison of ${ }^{12} \mathrm{CX}$ and ${ }^{13} \mathrm{C} X$ lines of similar intensity and excitation potential. A program of ${ }^{12} \mathrm{C} /{ }^{13} \mathrm{C}$ measurements is in progress based on the infrared spectra and near infrared photoelectric scans. Initial results for $\alpha$ Ori are described here.

The bandheads of both ${ }^{12} \mathrm{C}^{16} \mathrm{O}$ and ${ }^{13} \mathrm{C}^{16} \mathrm{O}$ molecules are prominent in even low resolution spectra of the $2.3 \mu$ first overtone bands. The problem is not in the identifi- 

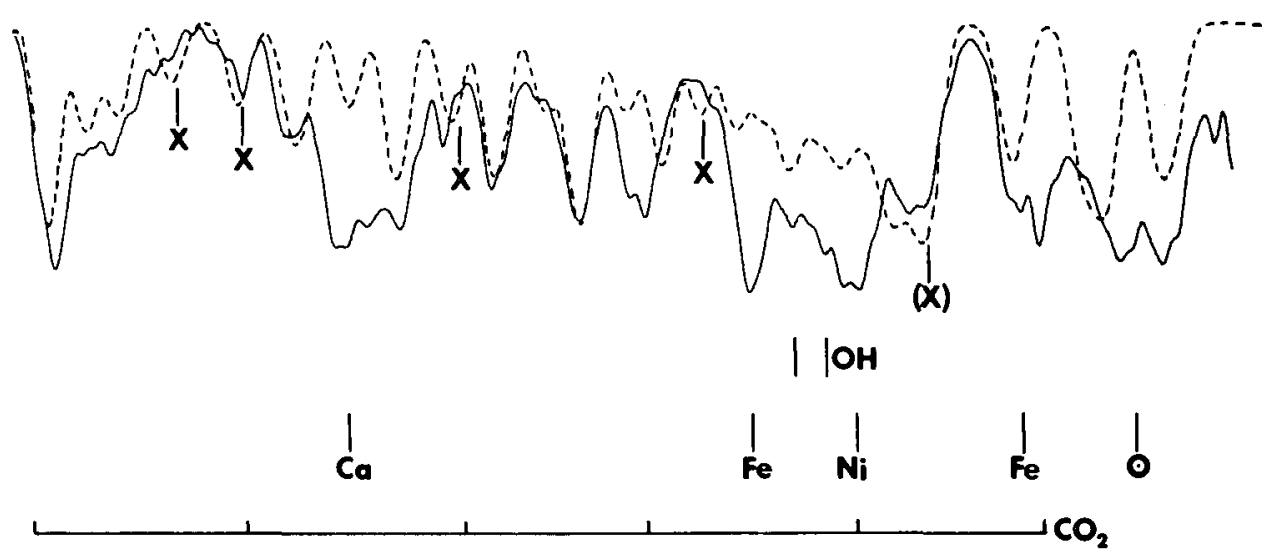

6195.0

6200.0

WAVENUMBER

Fig. 7. The $R$ branch head of the $4-1{ }^{13} \mathrm{C}^{16} \mathrm{O}$ vibration-rotation band in the spectrum of $\alpha$ Ori. The synthesized spectrum (dashed line) includes the ${ }^{12} \mathrm{C}^{16} \mathrm{O},{ }^{13} \mathrm{C}^{16} \mathrm{O},{ }^{12} \mathrm{CN}$ and ${ }^{13} \mathrm{CN}$ lines but not the $\mathrm{OH}$ and atomic lines identified below the spectrum. This synthesis assumes ${ }^{12} \mathrm{C} /{ }^{13} \mathrm{C}=5$ and the ${ }^{13} \mathrm{C}^{16} \mathrm{O}$ transitions are the dominant contributions to the regions labelled ' $X$ '.

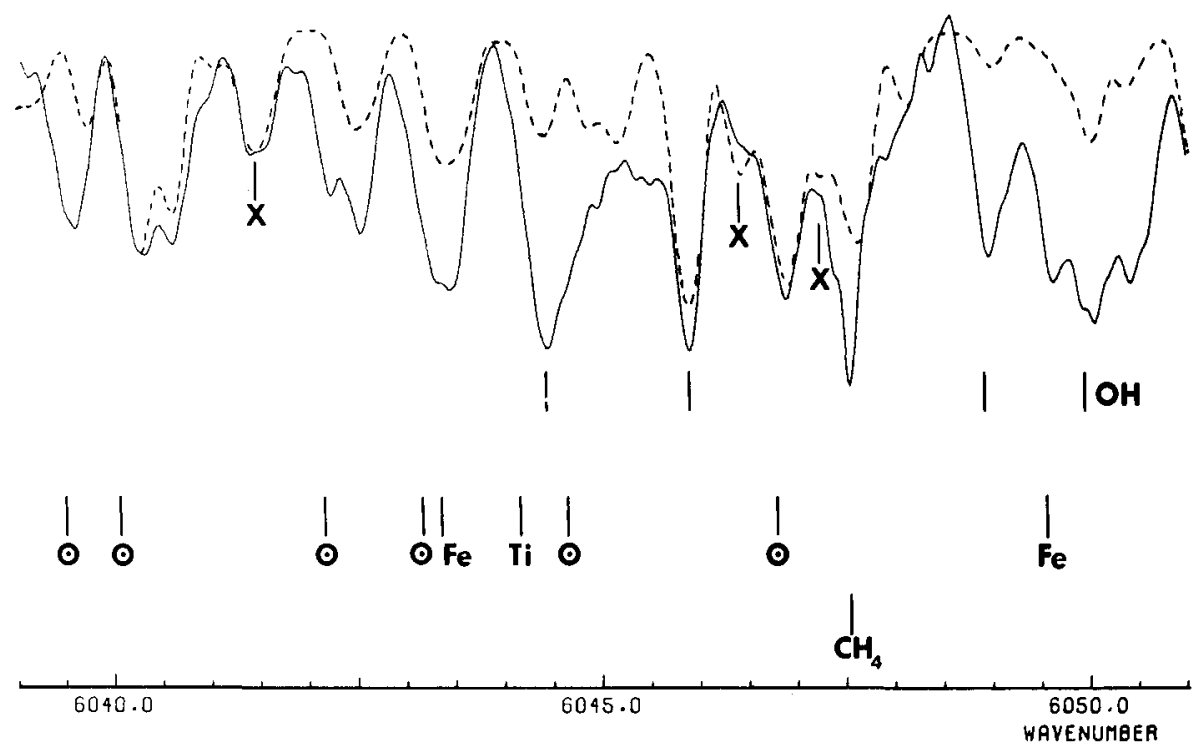

Fig. 8. The $R$ branch head of the $6-3{ }^{13} \mathrm{C}^{16} \mathrm{O}$ vibration-rotation band in the spectrum of $\alpha$ Ori. The synthesized spectrum (dashed line) includes only the ${ }^{12} \mathrm{C}^{16} \mathrm{O},{ }^{13} \mathrm{C}^{16} \mathrm{O},{ }^{12} \mathrm{CN}$ and ${ }^{13} \mathrm{CN}$ lines. This synthesis is for ${ }^{12} \mathrm{C} /{ }^{13} \mathrm{C}=6$ with ${ }^{13} \mathrm{C}^{16} \mathrm{O}$ transitions dominant in regions labelled ' $X$ '. 
cation but in the interpretation of these saturated lines. Weak lines are to be preferred for abundance estimates.

This discussion looks at the possibility of extracting the ${ }^{12} \mathrm{C} /{ }^{13} \mathrm{C}$ ratio from the weak second-overtone bands near $1.6 \mu$. The ${ }^{12} \mathrm{C}^{16} \mathrm{O}$ bandheads are moderately saturated but weak unsaturated lines can be identified (see Figure 1). The ${ }^{13} \mathrm{C}^{16} \mathrm{O}$ bandheads are visible but analysis is far from straight-forward on account of blending by atomic and molecular lines. At lower resolution, $\mathrm{a}^{12} \mathrm{C} /{ }^{13} \mathrm{C}$ analysis from this region would be very difficult. The 4-1 and 6-3 ${ }^{13} \mathrm{C}^{16} \mathrm{O}$ bandheads are illustrated in Figures 7 and 8. The predicted spectrum includes $\mathrm{CO}$ and $\mathrm{CN}$ lines but not the various atomic and $\mathrm{OH}$ lines which are identified on the figures. Telluric $\mathrm{CO}_{2}$ and $\mathrm{CH}_{4}$ lines are indicated.

The 4-1 bandhead is heavily blended. The region beyond the bandhead shows almost as much structure as the $\mathrm{CO}$ bandhead itself ! The predicted spectrum corresponds to a ratio ${ }^{12} \mathrm{C} /{ }^{13} \mathrm{C}=5$ and the regions marked by ' $X$ ' were given greatest weight in determining this ratio. In fact, a ratio ${ }^{12} \mathrm{C} /{ }^{13} \mathrm{C} \sim 6$ would provide a better fit. The region used in the derivation of the ${ }^{12} \mathrm{C} /{ }^{13} \mathrm{C}$ ratio extended beyond the portion illustrated in Figure 7.

The 6-3 bandhead is similarly confused by blends. The predicted spectrum with ${ }^{12} \mathrm{C} /$ ${ }^{13} \mathrm{C}=6$ provides a reasonable match to the observed spectrum. Other bands have been examined and confirm the results for the 4-1 and 6-3 bands. A study of possible sources of error is in progress; for example, the microturbulent velocity might be increased from the assumed $7 \mathrm{~km} \mathrm{~s}^{-1}$ to improve the fit to the observed spectrum. The effect on the ${ }^{12} \mathrm{C} /{ }^{13} \mathrm{C}$ ratio will be small because the ${ }^{13} \mathrm{C}^{16} \mathrm{O}$ lines are being compared with ${ }^{12} \mathrm{C} /{ }^{16} \mathrm{O}$ lines of very similar equivalent width. The result of the analysis is ${ }^{12} \mathrm{C} /$ ${ }^{13} \mathrm{C} \sim 6 \pm 2$ where the uncertainty is estimated from the deterioration of the fit to the observed spectrum.

The ${ }^{12} \mathrm{C} /{ }^{13} \mathrm{C}$ ratio may also be derived from the first-overtone band near $2.3 \mu$. special attention must be given to a correct treatment of saturation. One approach is to search for weak lines in these bands; members of the $P$ and $R$ branches with very low or very high rotational quantum number should be weak. Comparison of lines of equal intensity can be attempted. The non-linearity resulting from saturation was illustrated by Lambert and Dearborn (1972) in a discussion of the bands in $\alpha$ Boo. Analysis of these bands is underway and the initial sample of stars includes $\alpha$ Boo, $\alpha$ Her and $\alpha$ Sco.

Another approach to the ${ }^{12} \mathrm{C} /{ }^{13} \mathrm{C}$ ratio is to consider other molecules. The $2-0$ band of the $\mathrm{CN}$ red system near $8000 \AA$ is being observed with the Tull coudé scanner. Initial results of this program are described elsewhere (Day et al., 1973). A typical scan for the $\mathrm{CN}$ rich $\mathrm{K}$ giant $\alpha$ Ser is shown in Figure 9. The ${ }^{13} \mathrm{CN}$ lines are readily identifiable. A ratio ${ }^{12} \mathrm{C} /{ }^{13} \mathrm{C}=12 \pm 2$ is derived. Analysis of similar scans for $\alpha$ Boo gave ${ }^{12} \mathrm{C} /{ }^{13} \mathrm{C}=7.2 \pm 1.5$.

Scans of $\mathrm{CN}$ in $\alpha$ Ori were obtained. Figure 10 covers the same interval as Figure 9 and ${ }^{13} \mathrm{CN}$ lines in $\alpha$ Ori are clearly present in considerable strength. The extremely broad lines introduce great difficulties in locating the continuum from scans as short as the one illustrated in Figure 9. To alleviate this difficulty, scans at a resolution of 
$0.1 \AA$ and covering $30 \AA$ have been obtained. Analysis gives ${ }^{12} \mathrm{C} /{ }^{13} \mathrm{C} \sim 5$ to confirm the result from the $\mathrm{CO}$ second-overtone bands. A paper describing the $\mathrm{CO}$ and $\mathrm{CN}$ analyses is being completed for publication (Lambert et al., 1974).

The $\mathrm{CN}$ line intensities in $\alpha$ Ori show an odd behavior relative to the model atmosphere predictions. In the observed spectrum the $P, Q$ and $R^{12} \mathrm{CN}$ lines of similar rotational quantum number have very similar intensities with $Q$ branch lines appearing only slightly stronger than adjacent $P$ and $R$ lines. The model atmosphere predictions show the $Q$ lines to be markedly stronger than the $P$ and $R$ lines. This is the anticipated behavior based upon the relative oscillator strengths. The correct explanation is unknown. A possibility is that these molecular lines are formed by scattering. Then, the line source function, $S_{l}$, involves an integration over the radiation field which reduces to the mean continuum intensity, $S_{l}=J_{\lambda}^{c}$ in the limit where the atmosphere is optically thin at the center of the $\mathrm{CN}$ lines. The present calculations assume the lines are formed by pure absorption or $S_{l}=B_{\lambda}(T)$. Calculations show that at $8000 \AA$, the mean intensity $J_{\lambda}^{c}$ exceeds the Planck function $B_{\lambda}(T)$ and the gradient of $J_{\lambda}^{c}$ through the atmosphere is smaller than that for $B_{\lambda}(T)$. Since several vibrational bands can excite the upper state of the $2-0$ band, the appropriate mean intensity is a

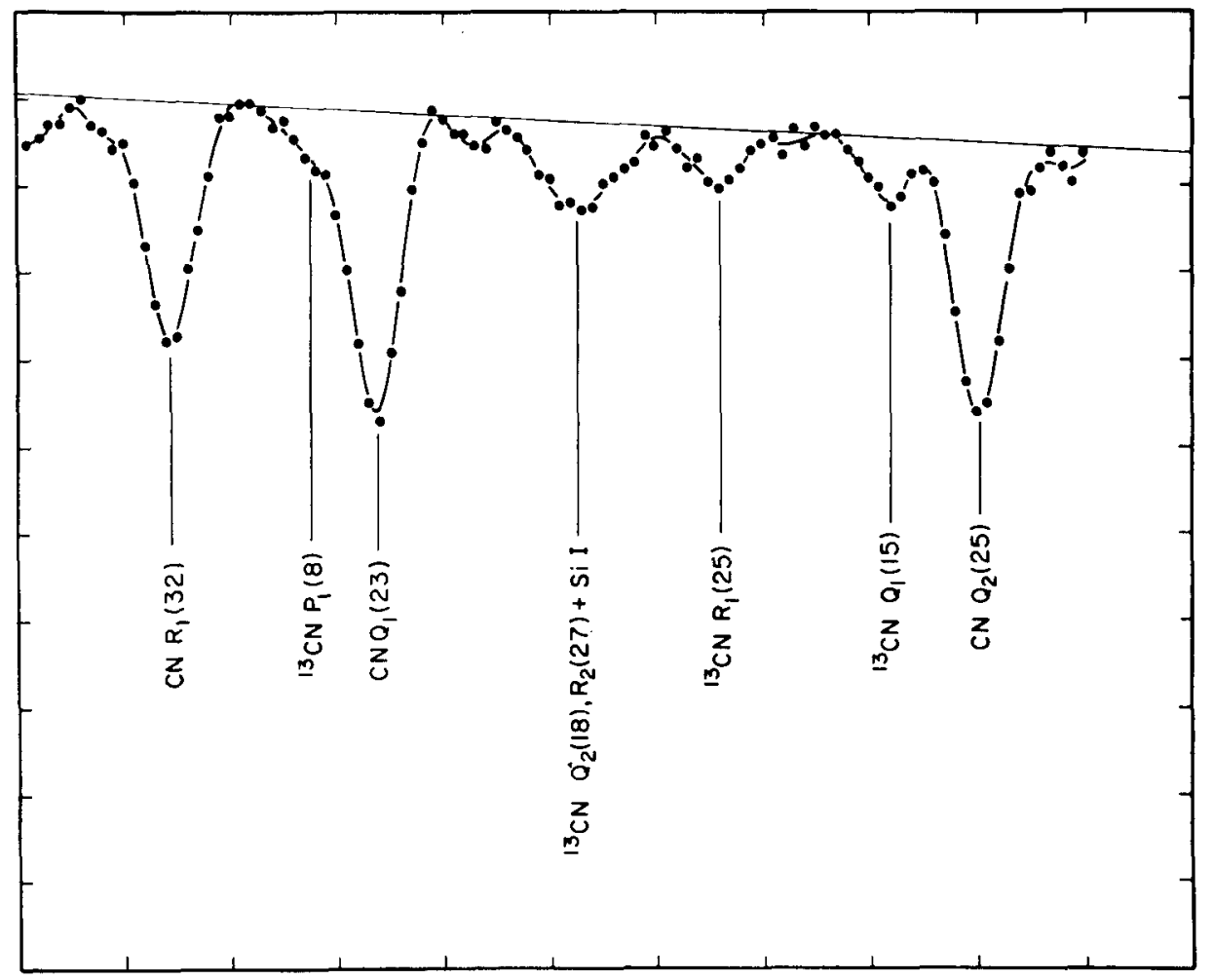

Fig. 9. A photoelectric scan of the spectrum of $\alpha$ Ser near $7975 \AA$. Four ${ }^{13} \mathrm{CN}$ features are prominent. The line ${ }^{13} \mathrm{CN} P_{1}(8)$ is blended with a ${ }^{12} \mathrm{CN}$ line. 


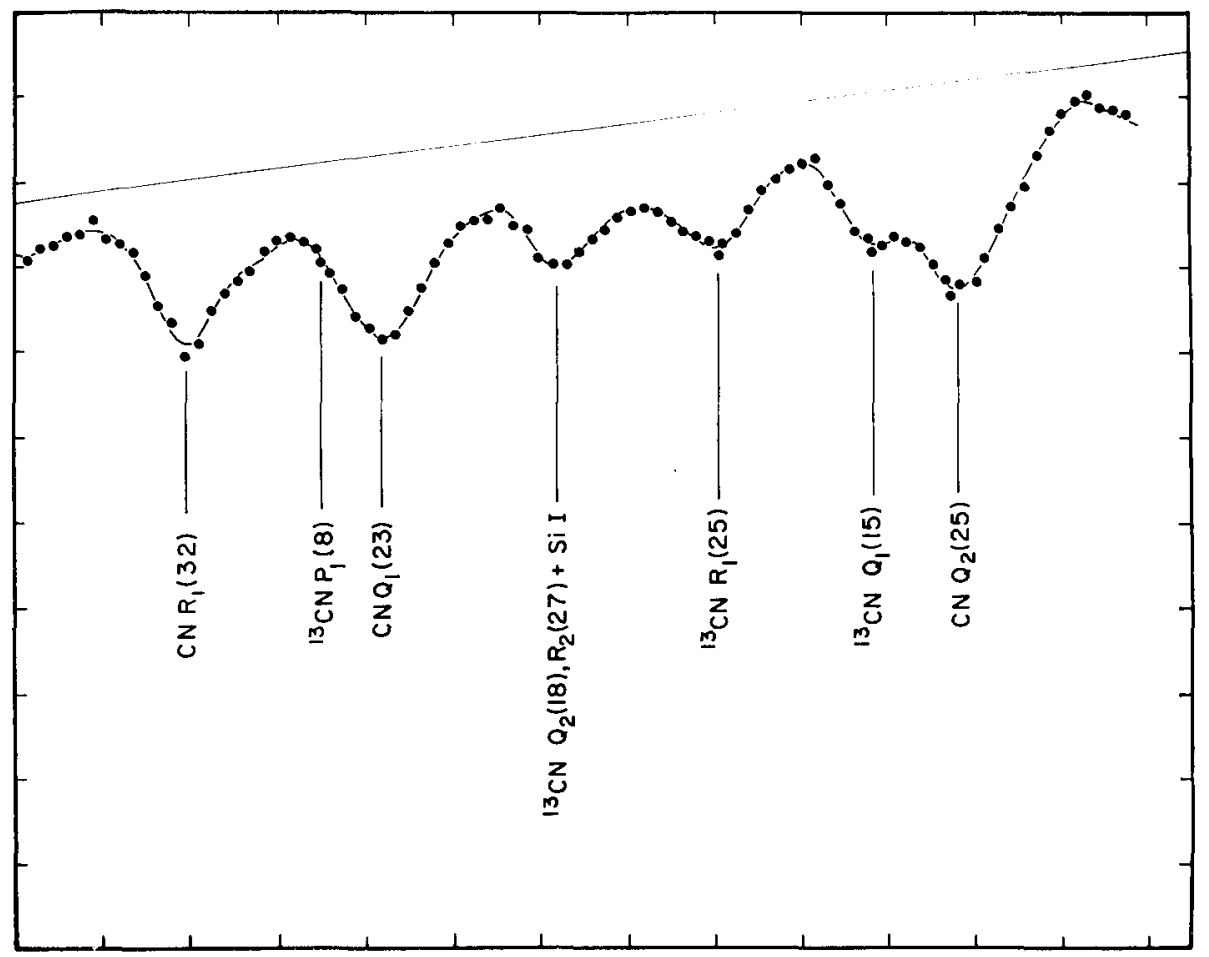

Fig. 10. A photoelectric scan of the spectrum of $\alpha$ Ori for the interval near $7975 \AA$. A comparison with Figure 9 shows that the ${ }^{13} \mathrm{CN}$ lines in the $\alpha$ Ser spectrum are readily identifiable in this scan of $\alpha$ Ori.

summation over mean intensities at several wavelengths. The difference between the mean $J_{\lambda}^{c}$ and $B_{\lambda}(T)$ persists when this summation is carried out. As $J_{\lambda}^{c}$ exceeds $B_{\lambda}(T)$, a line formed by scattering is weaker than the pure absorption equivalent and the shallower gradient of $J_{\lambda}^{c}$ results in smaller intensity differences between lines. While this idea provides a qualitative explanation, detailed calculations show that the $\mathrm{CN}$ lines in the present model atmosphere are formed in deep layers such that the mean intensity closely approaches the local Planck function. A general discussion of the appropriate source function for molecular lines in stellar atmospheres is given by Hinkle and Lambert (1974). The scattering hypothesis and alternative explanations for the CN intensity anomaly are described by Lambert $e t$ al. (1974).

\section{The ${ }^{16} \mathrm{O} /{ }^{18} \mathrm{O}$ and ${ }^{16} \mathrm{O} /{ }^{17} \mathrm{O}$ Abundance Ratios}

The spectrum of $\alpha$ Ori in the region of the $\mathrm{CO}$ fundamental lines has been examined for evidence of the oxygen isotopes ${ }^{17} \mathrm{O}$ and ${ }^{18} \mathrm{O}$. The terrestrial ratios are ${ }^{16} \mathrm{O} /{ }^{17} \mathrm{O}=$ $=2700$ and ${ }^{16} \mathrm{O} /{ }^{18} \mathrm{O}=490$. The column density of $\mathrm{CO}$ is sufficiently large that many ${ }^{12} \mathrm{C}^{16} \mathrm{O}$ and ${ }^{13} \mathrm{C}^{16} \mathrm{O}$ are highly saturated and detection of ${ }^{12} \mathrm{C}^{18} \mathrm{O}$ and even ${ }^{13} \mathrm{C}^{18} \mathrm{O}$ lines can be anticipated (Lambert, 1973). Adverse consequences of the considerable 
saturation are readily apparent: the spectrum is extremely rich in ${ }^{12} \mathrm{C}^{16} \mathrm{O}$ and ${ }^{13} \mathrm{C}^{16} \mathrm{O}$ lines and the extraction of an abundance ratio from a line intensity ratio must be performed with caution. Regions free from ${ }^{12} \mathrm{C}^{16} \mathrm{O}$ and ${ }^{13} \mathrm{C}^{16} \mathrm{O}$ lines are rare. In addition, the rotational structure is developed in the stellar spectrum beyond the point to which the available molecular constants can be used to predict accurate wavenumbers. This introduces the possibility that lines attributed to rare isotopes are merely unrecognized ${ }^{12} \mathrm{C}^{16} \mathrm{O}$ or ${ }^{13} \mathrm{C}^{16} \mathrm{O}$ lines.

The $5 \mu$ spectrum of $\alpha$ Ori was searched for ${ }^{12} \mathrm{C}^{17} \mathrm{O}$ and ${ }^{12} \mathrm{C}^{18} \mathrm{O}$ lines. ${ }^{12} \mathrm{C}^{18} \mathrm{O}$ was positively identified through about 10 well resolved lines. The rich spectrum demands an analysis by spectrum synthesis techniques. A segment of the spectrum is illustrated in Figure 11. The well resolved ${ }^{12} \mathrm{C}^{18} \mathrm{O}$ lines at $1977.5 \mathrm{~cm}^{-1}$ is the $P(20) 1-0$ transition. The predicted spectrum (broken line) has been multiplied by the transmission of the Earth's atmosphere. The predictions were made for ${ }^{16} \mathrm{O} /{ }^{18} \mathrm{O}=490$ and $\infty\left(\right.$ no ${ }^{18} \mathrm{O}$ present). The stellar abundance ratio ${ }^{16} \mathrm{O} /{ }^{18} \mathrm{O}$ is considered equal to the terrestrial

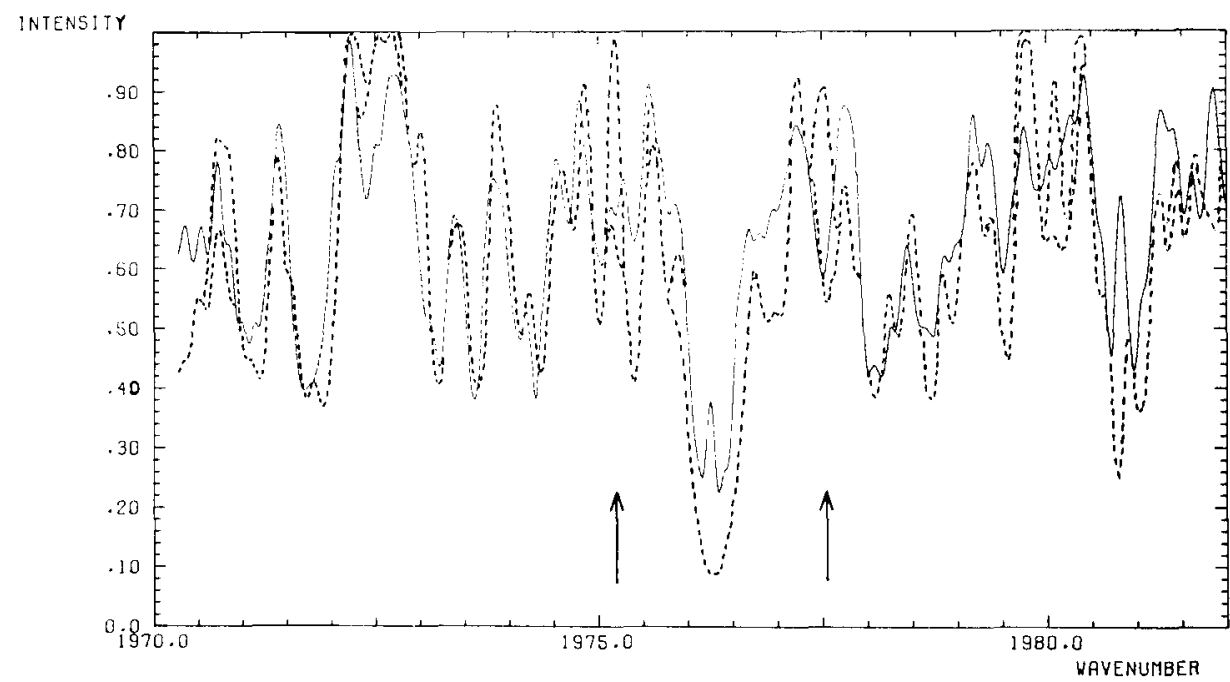

Fig. 11. The spectrum of $\alpha$ Ori between 1970 and $1982 \mathrm{~cm}^{-1}$. The synthesized spectra (dashed lines) include all CO lines; the lower and upper traces corresponds to ratios ${ }^{16} \mathrm{O} /{ }^{18} \mathrm{O}=480$ and $\infty$ respectively. The upper trace is coincident with the lower trace except in regions where ${ }^{12} \mathrm{C}^{18} \mathrm{O}$ or ${ }^{13} \mathrm{C}^{18} \mathrm{O}$ provides a dominant contribution and two such prominent regions are identified by the arrows.

ratio within an uncertainty of a factor of 3 . The ${ }^{12} \mathrm{C}^{17} \mathrm{O}$ molecule cannot be identified. Analysis shows that the observed spectrum is not inconsistent with a terrestrial ${ }^{16} \mathrm{O} /{ }^{17} \mathrm{O}$ ratio; the strongest ${ }^{12} \mathrm{C}^{17} \mathrm{O}$ lines are severely blended.

A careful comparison of predicted and observed spectra reveals some interesting discrepancies. A few unidentified stellar lines are present. Some CO lines appear weaker than predicted. This is possibly attributable to departures from local thermodynamic equilibrium. A study of collision rates (Thompson, 1973; Hinkle and Lambert, 1974) shows that rotational states for a vibrational level should be kept in 
equilibrium while vibrational levels may show departures. Tentative evidence from the systematics of the discrepant CO lines supports this conclusion.

Interpretation of fundamental $\mathrm{CO}$ lines is difficult. According to the present model atmosphere, the ${ }^{12} \mathrm{C}^{18} \mathrm{O}$ lines are not too seriously affected by saturation. Saturation is very severe for slightly stronger lines as the following numerical result illustrates: a typical ${ }^{12} \mathrm{C}^{16} \mathrm{O}$ fundamental line may have a predicted equivalent width $W_{\sigma}=0.3$ $\mathrm{cm}^{-1}$ and the identical line from an isotopic species reduced in abundance by a factor of 500 has $W_{\sigma}=0.15 \mathrm{~cm}^{-1}$, i.e. reduction by only one-half. The boundary temperature for the atmosphere effectively determines the value of $W_{\sigma}$ for which saturation begins to be severe; a high boundary temperature can produce weak lines which are strongly saturated. The risk of deducing incorrect isotopic abundance ratios may be minimized by comparing lines of similar intensity and weak lines should be used whenever possible. A possible approach would be to compare ${ }^{12} \mathrm{C}^{18} \mathrm{O}$ fundamental lines with the weak ${ }^{12} \mathrm{C}^{16} \mathrm{O}$ second-overtone lines.

Another approach is to search for isotopic lines among the first-overtone $\mathrm{CO}$ lines. This was attempted for Arcturus. A high ratio ${ }^{16} \mathrm{O} /{ }^{18} \mathrm{O} \sim 40$ was indicated by Geballe et al. (1972) in an analysis of a high resolution scan for a small interval of the
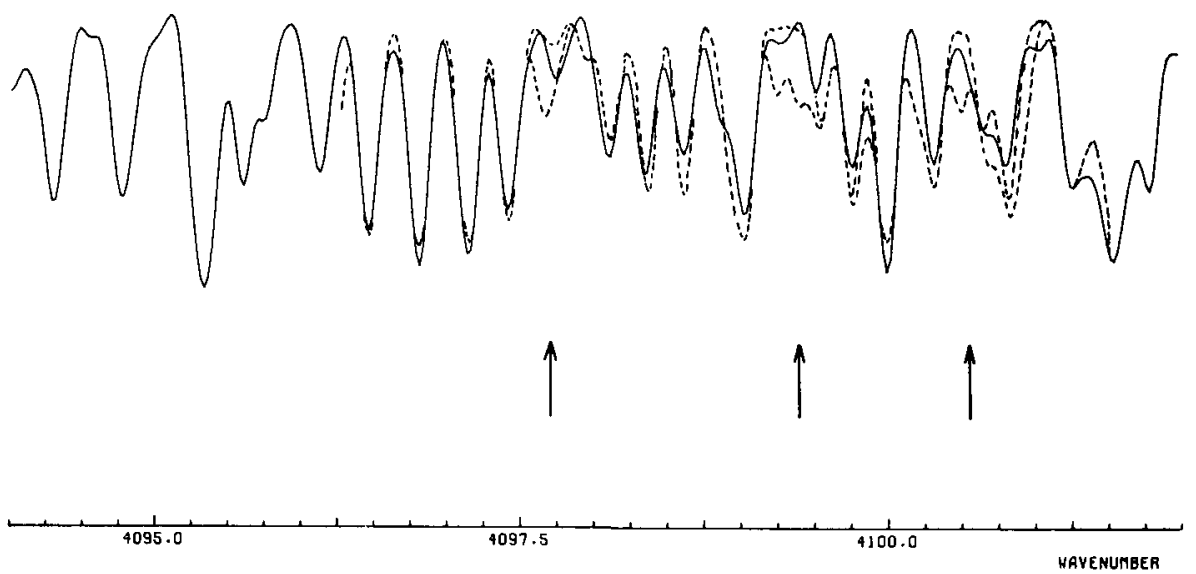

Fig. 12. The spectrum of $\alpha$ Boo between 4093 and $4104 \mathrm{~cm}^{-1}$. The synthesized spectra (dashed lines) include only $\mathrm{CO}$ lines; the lower and upper traces correspond to high $\left({ }^{16} \mathrm{O} /{ }^{18} \mathrm{O}=40\right)$ and normal $\left({ }^{16} \mathrm{O} /{ }^{18} \mathrm{O}=480\right)$ abundances of ${ }^{18} \mathrm{O}$. The arrows identify regions in which the observed ${ }^{12} \mathrm{C}^{18} \mathrm{O}$ contribution is incompatible with the predicted spectrum for ${ }^{16} \mathrm{O} /{ }^{18} \mathrm{O}=40$.

$\mathrm{CO}$ fundamental region. Model atmosphere calculations showed that ${ }^{12} \mathrm{C}^{18} \mathrm{O}$ lines should be readily visible in the first-overtone region for ${ }^{16} \mathrm{O} /{ }^{18} \mathrm{O} \sim 40$. A thorough analysis of the observed spectrum reveals no acceptable identifications for ${ }^{12} \mathrm{C}^{18} \mathrm{O}$ or ${ }^{12} \mathrm{C}^{17} \mathrm{O}$ lines. The lack of ${ }^{12} \mathrm{C}^{18} \mathrm{O}$ lines is well shown in Figure 2. The predicted spectra (dashed line) correspond to ${ }^{16} \mathrm{O} /{ }^{18} \mathrm{O}=40$ and ${ }^{16} \mathrm{O} /{ }^{18} \mathrm{O}=490$ (terrestrial). Several excellent regions are available to provide a firm lower limit to the ${ }^{16} \mathrm{O} /{ }^{18} \mathrm{O}$ ratio: the preliminary estimate is ${ }^{16} \mathrm{O} /{ }^{18} \mathrm{O} \gtrsim 300$. A similar result is obtained for the ${ }^{16} \mathrm{O} /{ }^{17} \mathrm{O}$ ratio. 
This spectral region in stars with a larger column density of $\mathrm{CO}$ can be expected to provide positive identifications for both ${ }^{17} \mathrm{O}$ and ${ }^{18} \mathrm{O}$. The $\mathrm{M}$ supergiants show considerably stronger $\mathrm{CO}$ both lines in rough accord with predictions. A difficulty is that some supergiants have very broad lines and many ${ }^{12} \mathrm{C}^{18} \mathrm{O}$ lines will be unresolved. The ${ }^{12} \mathrm{C}^{17} \mathrm{O}$ isotope is more favorably placed in that part of the $2-0 \mathrm{R}$ branch between 4280 and $4295 \mathrm{~cm}^{-1}$ is in a region containing a minimum of ${ }^{12} \mathrm{C}^{16} \mathrm{O}$ lines and no ${ }^{13} \mathrm{C}^{16} \mathrm{O}$ lines. Figure 13 shows how a part of this region appears in Arcturus. In the spectrum of $\alpha \mathrm{Her}$, relatively strong lines appear at the positions of the ${ }^{12} \mathrm{C}^{17} \mathrm{O}$ lines: $9 R$ branch lines between $J=20$ and $J=32$ are well resolved. By happy circumstance, the favorably located part of the 2-0 band contains the intensity maximum in the $R$

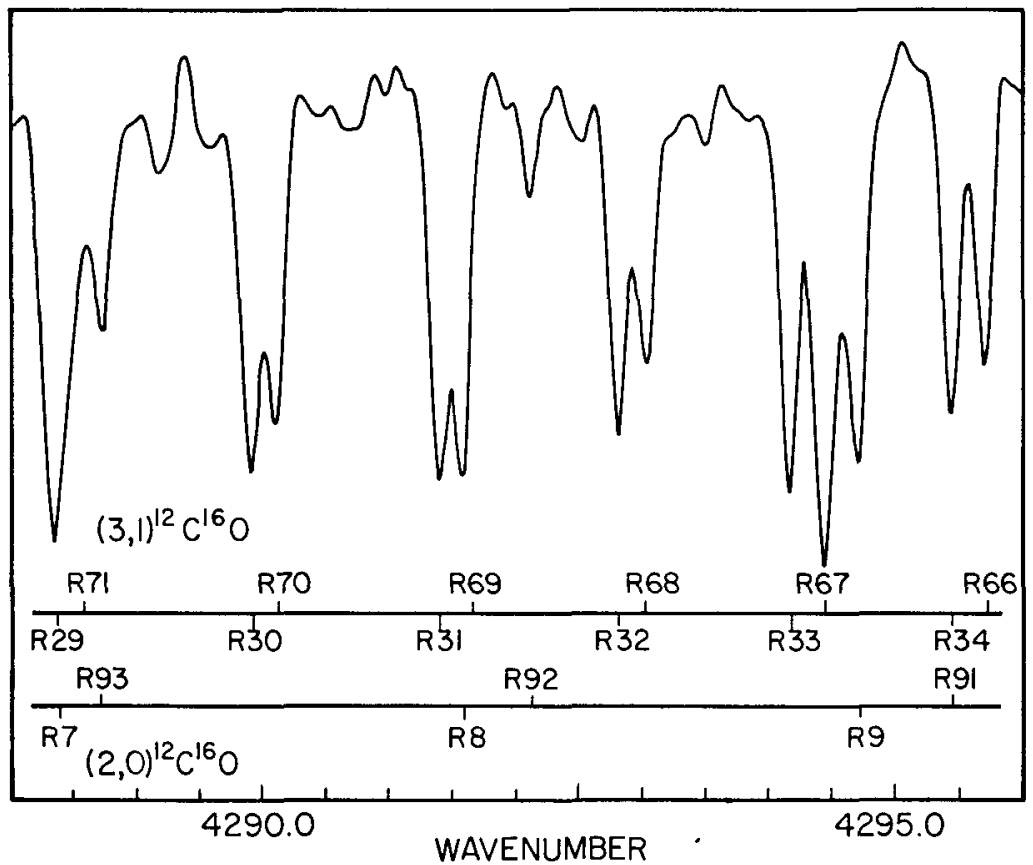

Fig. 13. The spectrum of $\alpha$ Boo between 4288 and $4296 \mathrm{~cm}^{-1} \cdot{ }^{12} \mathrm{C}^{16} \mathrm{O}$ lines are identified. The 2-0 $R$ branch ${ }^{12} \mathrm{C}^{17} \mathrm{O}$ lines are located across this and neighboring regions. The relatively low density of ${ }^{12} \mathrm{C}^{16} \mathrm{O}$ lines and the absence of ${ }^{13} \mathrm{C}^{16} \mathrm{O}$ lines eases the detection of weaker ${ }^{12} \mathrm{C}^{17} \mathrm{O}$ lines. In this particular region, the ${ }^{12} \mathrm{C}^{17} \mathrm{O}$ lines with the exception of $R(32)$ at $4291.8 \mathrm{~cm}^{-1}$ are blended with stronger ${ }^{12} \mathrm{C}^{16} \mathrm{O}$ lines.

branch. A few other ${ }^{12} \mathrm{C}^{17} \mathrm{O}$ lines in other vibrational bands have been identified. The ${ }^{12} \mathrm{C}^{18} \mathrm{O}$ lines are not so favorably placed but a few weak lines can be identified. A simple analysis suggests ${ }^{17} \mathrm{O} /{ }^{18} \mathrm{O}>1$ which is to be compared with the terrestrial ratio $\simeq \frac{1}{5}$. The relative overabundance of ${ }^{17} \mathrm{O}$ can be linked to the CNO-cycle. These identifications confirm the results reported by Maillard (1973) who presents a more extensive abundance analysis. 


\section{M Stars and SiO Vibration-Rotation Bands}

The fundamental vibration-rotation bands for $\mathrm{SiO}$ are located at about $8 \mu$. The first-overtone bands near $4 \mu$ were discovered by Cudaback et al. (1971) in spectra of $\alpha$ Ori at a resolution of $2 \mathrm{~cm}^{-1}$. The individual rotational lines can be identified away from the bandhead in the $0.1 \mathrm{~cm}^{-1}$ resolution spectra of $\alpha$ Ori. Indeed, these spectra provided a sufficient number of identified lines with accurate line positions that the molecular constants for the $\mathrm{SiO}$ electronic ground state were improved (Beer et al., 1973). The strength of the SiO lines in $\alpha$ Ori is approximately consistent with model atmosphere calculations, abundance expectations and the transition probabilities computed by Hedelund and Lambert (1972).

The SiO column density is predicted to increase for later spectral types. $\mathrm{SiO}$ is detected for $\alpha$ Her but the bandheads are absent from spectra of the Mira variables $o$ Cet and R Leo obtained at several phases. This absence is not in accord with expectation.

Several possible explanations are under consideration: abundance adjustments, depletion of $\mathrm{SiO}$ by grain formation, atmospheric structure peculiarities (i.e. a flat temperature profile), and dilution of the photospheric $4 \mu$ spectrum by continuum radiation from the circumstellar dust shell.

\section{The $\mathrm{H}_{2}$ Quadrupole Vibration-Rotation Lines}

Although $\mathrm{H}_{2}$ is an abundant molecule in cool stars, this symmetric molecule does not have an electric dipole vibration-rotation spectrum. Weak quadrupole transitions do exist and have been detected in the spectra of the major planets. Spinrad (1966) identified two $\mathbf{H}_{2}$ 2-0 quadrupole lines in spectra of the sequence of $\mathrm{M}$ stars from $\alpha$ Ori to $o$ Cet. Later, it was noted that stronger 1-0 lines were not seen in a high resolution spectrum of $\alpha$ Ori (Spinrad and Wing, 1969). Convincing explanations for this discrepancy were unavailable (Spinrad, 1973) and a reexamination was undertaken.

A search for 1-0 lines was made using interferometric spectra of $\alpha$ Ori, $\alpha$ Sio, $\alpha$ Her, $\mathrm{R}$ Leo, $o$ Cet and W Hya. Tentative identifications were established for 3 lines in the $\alpha$ Her spectra and upper limits were set for all other stars. These measurements are consistent with the model atmosphere predictions provided by Goon and Auman (1970). These results are at odds with the presence of 2-0 lines in these stars. The 2-0 identifications were examined and alternative identifications were proposed. A full discussion appears in a paper by Lambert et al. (1973).

\section{Summary}

In this paper, an attempt has been made to display the diverse contributions of high resolution infrared spectroscopy to a fuller understanding of the physics of cool stars.

The important conclusions of a general nature appear to be: 
- infrared spectra are rich in information: the illustrations given here can be supplemented by radial velocity studies and detailed analyses of line profiles.

- the infrared spectra should not be considered in isolation: other spectral regions contain essential information, i.e. the $\left[\mathrm{C}_{\mathrm{I}}\right]$ and $\left[\mathrm{O}_{\mathrm{I}}\right]$ lines should be included in an abundance analysis.

-. laboratory and theoretical studies on atoms and molecules are needed in order to extend available information on the wavelengths and intensities to provide a complete coverage for the spectrum analyses.

\section{Acknowledgements}

The active collaboration of the author's colleagues is deeply appreciated: important contributions have been made by Drs R. Beer, T. G. Barnes, A. L. Brooke and C. Sneden and Messrs. D. Dearborn and A. Bernat.

This paper presents one phase of activity in the joint Jet Propulsion LaboratoryUniversity of Texas Infrared Astronomy Program supported, in part, by National Science Foundation Grant GP-32322X with the University of Texas at Austin, and, in part, by National Aeronautics and Space Administration Contract NAS 7-100 with the Jet Propulsion Laboratory, California Institute of Technology.

\section{References}

Arnold, J. O. and Nicholls, R. W.: 1972, J. Quant. Spectrosc. Radiat. Transfer 12, 1435.

Arnold, J. O. and Nicholls, R. W.: 1973, J. Quant. Spectrosc. Radiat. Transfer 13, 115.

Beer, R., Barnes, T. G., and Lambert, D. L.: 1973, Proc. Conf. on Red Giant Stars, Indiana Univ.

Press., p. 84.

Beer, R., Hutchison, R. B., Norton, R. H., and Lambert, D. L.: 1972, Astrophys. J. 172, 89.

Beer, R., Norton, R. H., and Seaman, C. H.: 1971, Rev. Sci. Instr. 42, 1393.

Cudaback, D. D., Gaustad, J. E., and Knacke, R. F.: 1971, Astrophys. J. Letters 166, L490.

Day, R. W., Lambert, D. L., and Sneden, C.: 1973, Astrophys. J. 185, 213.

D'Incan, J., Effatin, C., and Roux, F.: 1971, J. Quant. Spectrosc. Radiat. Transfer 11, 1215.

Faÿ, T. D. and Johnson, H. R.: 1973, Astrophys. J. 181, 851.

Geballe, T. R., Wollman, E. R., and Rank, D. M.: 1972, Astrophys. J. Letters 177, L27.

Goon, G. and Auman, J. R.: 1970, Astrophys. J. 161, 533.

Hedelund, J. and Lambert, D. L.: 1972, Astrophys. J. Letters 11, 71.

Hinkle, K. H. and Lambert, D. L.: 1973, in preparation.

Lambert, D. L.: 1973, Proc. Conf. on Red Giant Stars, Indiana Univ. Press, p. 350.

Lambert, D. L. and Dearborn, D. S.: 1972, Mem. Soc. Roy. Sci Liège, 6th Ser. 3, 147.

Lambert, D. L. and Mallia, E. A.: 1974, Adv. Astron. Astrophys., in preparation.

Lambert, D. L., Brooke, A. L., and Barnes, T. G.: 1973, Astrophys. J. 186, 513.

Lambert, D. L., Dearborn, D. S., and Sneden, C. A.: 1974, in preparation.

Maillard, J. P.: 1973, this volume, p. 269.

Murphy, R. E.: 1971, J. Chem. Phys. 54, 4852.

Spinrad, H.: 1966, Astrophys. J. 145, 195.

Spinrad, H.: 1973, Proc. Conf. on Red Giant Stars, Indiana Univ. Press, p. 9.

Spinrad, H. and Wing, R. F.: 1969, Ann. Rev. Astron. Astrophys. 7, 249.

Spinrad, H., Kaplan, L. D., Connes, P., Connes, J., Kunde, V. G., and Maillard, J. P.: 1970 in G. W. Lockwood and H. M. Dyck (eds.), Proc. Conf. on Late-Type Stars, KPNO Contribution, No. 54, Tucson, Arizona. 
Thompson, R. I.: 1973, Astrophys. J. 181, 1039.

Tull, R. G.: 1972, 'Auxiliary Instrumentation for large Telescopes', in S. Laustsen and A. Reiz (eds.)

Proceedings of ESO/CERN Conference of May 1972, Geneva.

Young, L. A. and Eachus, W. J.: 1966, J. Chem. Phys. 44, 4195.

\section{DISCUSSION}

Underhill: I notice you said in fitting some of your lines you used a microturbulent velocity of the order of $10 \mathrm{~km} \mathrm{~s}^{-1}$. This seems hardly the thing to do because that would be supersonic at the temperature of your atmosphere. Supersonic microturbulence is a no-no.

Lambert: I don't think it is actually supersonic.

Underhill: What temperatures are you using?

Lambert: I suppose between about 2000 and $3000 \mathrm{deg}$.

Underhill: Well the velocity of your hydrogen line is far under $10 \mathrm{~km} \mathrm{~s}^{-1}$ at $3000 \mathrm{deg}$.

Lambert: There is a problem which I am trying to resolve at the present time, which is that the lines are definitely broad - of that there is no doubt - and some velocity field is broadening them; the question is whether you want to call it micro or macro turbulence.

Underhill: It makes a difference in computing your spectrum.

Lambert: When the lines are separate, yes I realize that.

Underhill: It throws suspicion on your methods of computing synthetic spectra.

Lambert: Well, we have to make a detailed comparison of line shapes. I have some very nice profiles of some well isolated atomic lines around one micron to do this sort of thing.

Hyland: Those synthetic spectra look very good and seem to fit beautifully but can you tell me how the model atmosphere calculations compare with the overall continuous energy distribution of the stars.

Lambert: It compares very well. This is described in a paper by Hollis Johnson in the Astrophysical Journal.

Hyland: Personally I didn't think they fitted very well.

Lambert: I did. I think the point is that the model atmosphere may not be too crucial because when you are talking about isotopic abundace ratios you are comparing ${ }^{12} \mathrm{C} /{ }^{16} \mathrm{O}$ lines with ${ }^{13} \mathrm{C} /{ }^{16} \mathrm{O}$ lines of about the same intensity and in the same wavelength region.

Hyland: That is all right when you are talking about isotopic abundances but when you are comparing the carbon and hydrogen abundance, that does not apply.

Lambert: Yes you are right. 